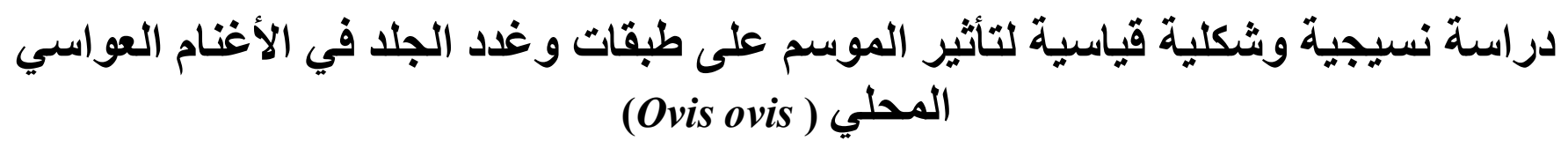

$$
\begin{aligned}
& \text { احمد نزار عبد الجواد الطائي } \\
& \text { فرع التشريح، كلية الطب البيطري، جامعة الموصل، الموصل، العراق }
\end{aligned}
$$

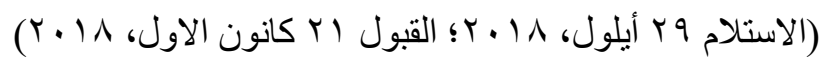

هدف الدراسة هو معرفة تأثثر الموسم على الجلد والغدد الجلدية في الأغنام العواسي المحلية وتم إجر ائها على خمسة أكباش عواسية

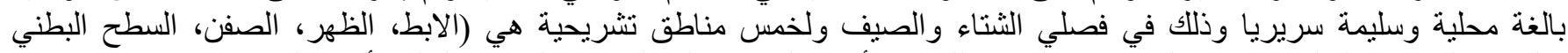

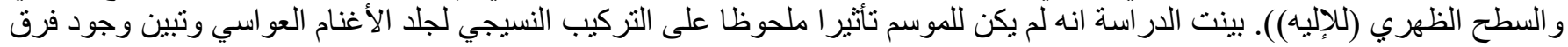

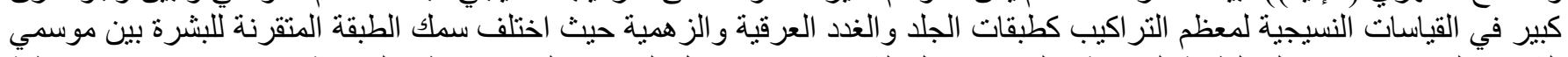

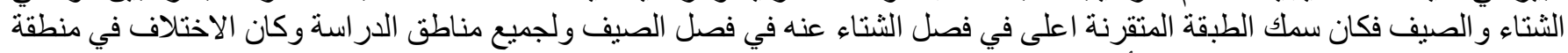

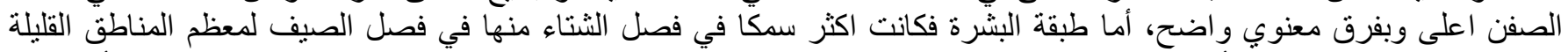

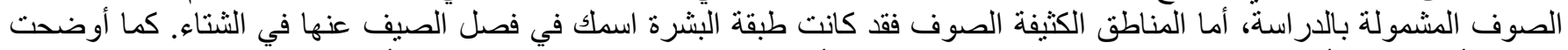

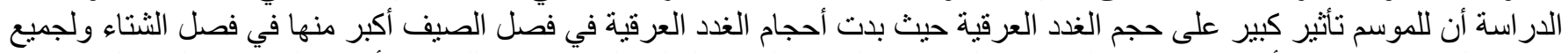

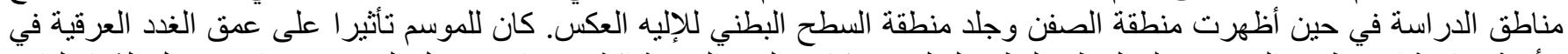

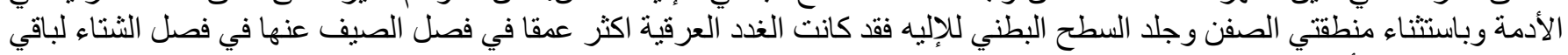

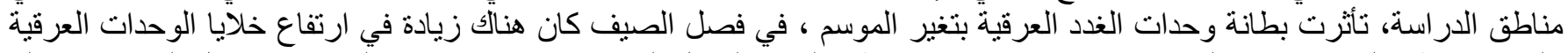

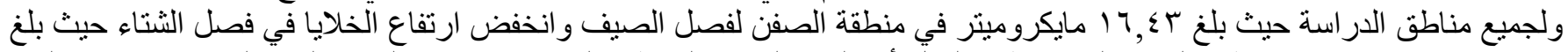

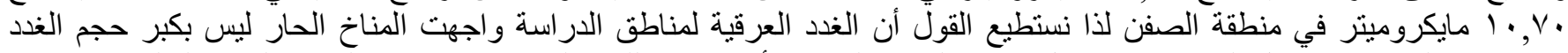

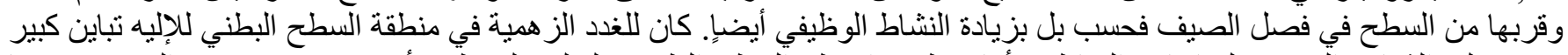

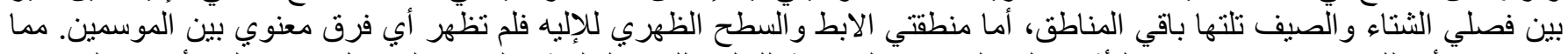

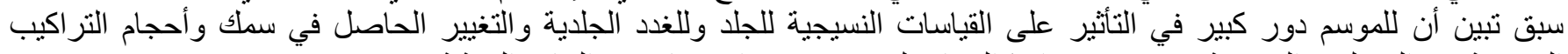
النسيجية بين الفصلين، التوصية بتحديد موسم اخذ العينات لتلافي حدوث التئين اختلافات في النتائج البحثية.

\title{
Histological and morphometrical study of the effect of season on layers and glands of skin in local Awassi sheep (Ovis ovis)
}

\author{
A.N. Abduljawaad \\ Department of Anatomy, College Veterinary Medicine, University of Mosul, Mosul, Iraq, ahmednazar99@yahoo.com
}

\begin{abstract}
The study aimed to investigate the effect of season on the skin and cutaneous glands in local Awassi breed of sheep (Ovis ovis). The study was done on five healthy adult rams of Awassi breed in winter and summer at five anatomical regions (armpit, back, scrotum, ventral surface of fatty tail and dorsal surface of the fatty tail). The study explained that season had no significant effect on the histological structure of the sheep's skin, while there was a significant variation in the histological measurements of most structures of skin such as skin layers, sweat and sebaceous glands where the thickness of the corneal layer of the skin varied between the winter and summer seasons, it was thicker in the winter than in the summer for most of the studied areas, the variation was higher and more significantly at scrotum, where the epidermis was thicker in winter than in
\end{abstract}


summer for most of the few wool areas that included in the study, while in the dense wool areas the epidermis was thicker in summer than in winter. The study also showed that the season has a significant effect on the size of the sweat glands. The size of the sweat glands in the summer appeared to be greater than in the winter and for all areas of study, but the area of the scrotum and the skin of the ventral surface of fatty tail showed the opposite. the season had an effect on the depth of the sweat glands in the dermis. With the exception of the scrotum and the ventral surface of the fatty tail, the sweat glands were deeper in the summer than in the winter for the rest of the study areas. The lining of the sweat glands units was affected by the change of season. In the summer, the height of the cells of the sweat units was increased and for all regions of the study to reached 16.43 micrometers in the scrotum area for the summer while decreased in winter to reached 10.70 micrometers. So, we can say that the sweat glands of the study areas faced the warm climate not only by the increase in the size of the glands and their proximity to the surface in the summer but also by increased functional activity. The sebaceous glands in the area of the ventral surface of the fatty tail had a large difference between the winter and summer followed by the rest of the areas, either the armpit areas and the dorsal surface of the fatty tail did not show any significant difference between the two seasons. Finally, we found the season has been shown to play a significant role in influencing the histological measurements of skin and skin glands.

Keyword: Skin, effect of season, Skin gland, Ovis ovis

Available online at http://www.vetmedmosul.com

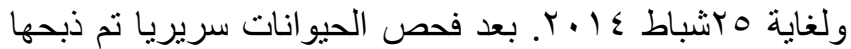

المقدمة

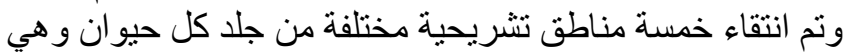
الابط، الصفن، الظهر، السطح الظهري و البطني للإليه. ثنتئت

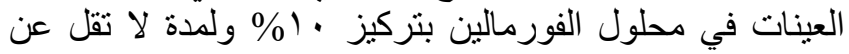

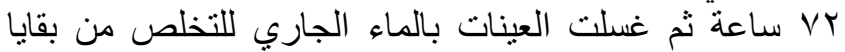

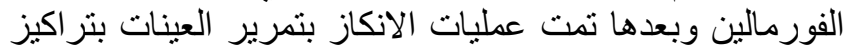

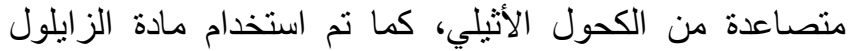

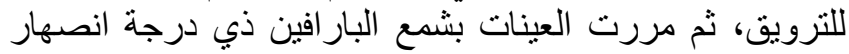

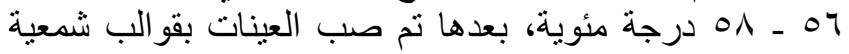

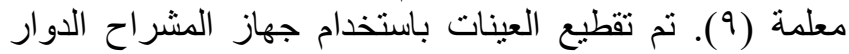

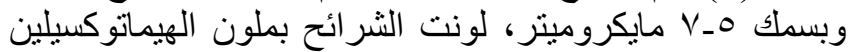

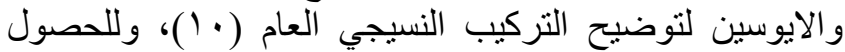

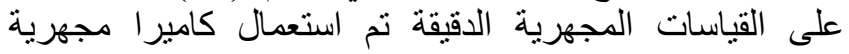

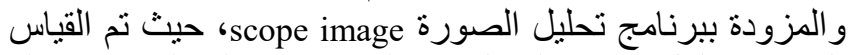

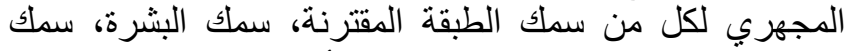

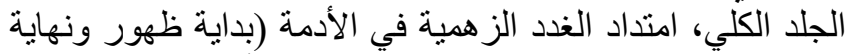

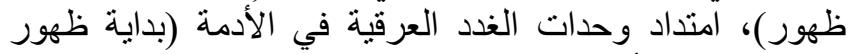

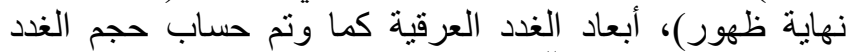
العرقية من خلال نق بُ ×

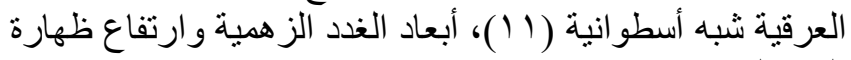

للجلد و غدده العرقية و الزهمية أهمية كبيرة في تتظيم حرارة

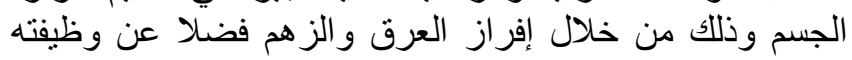

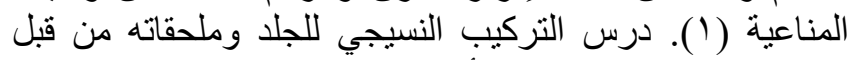

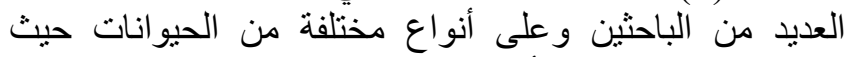

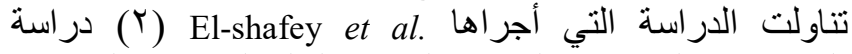

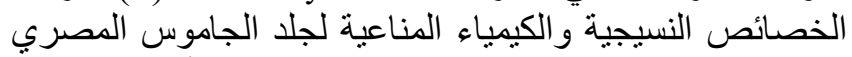

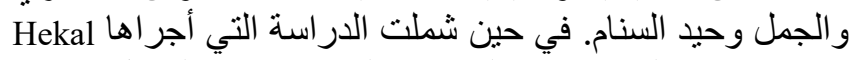

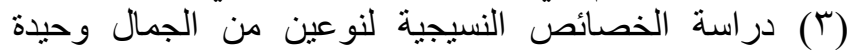

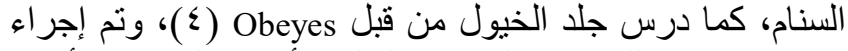

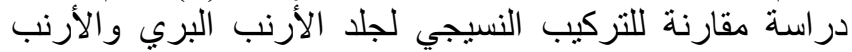

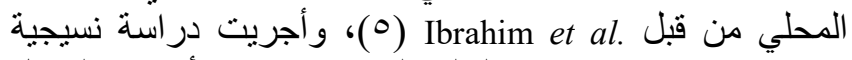

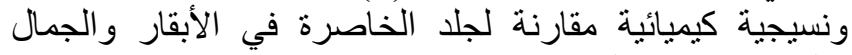

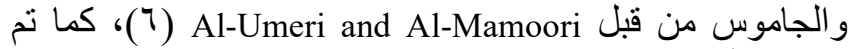
دراسة تأثير العمر والجنس على التى جلود الحيو الحيوانات في دراسات

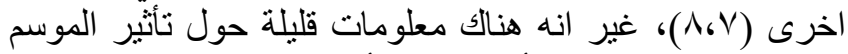

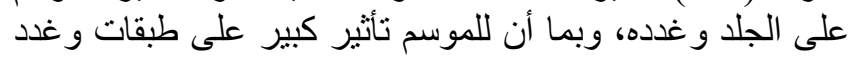

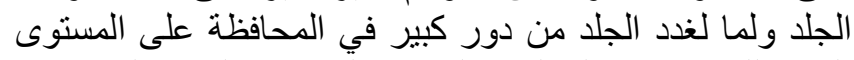

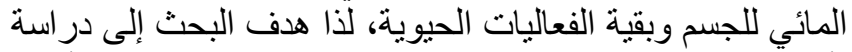

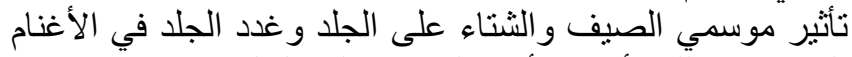

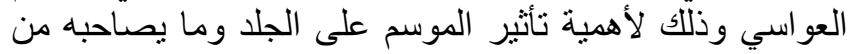

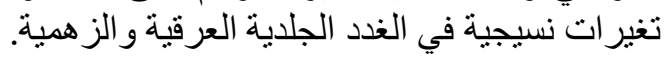

يتكون جلد الأغنام العواسي من طبقتين أساسيتين هما البشرة

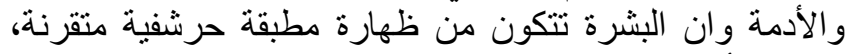

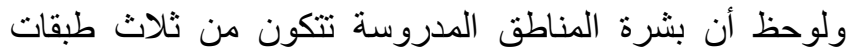

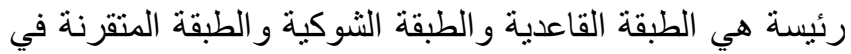

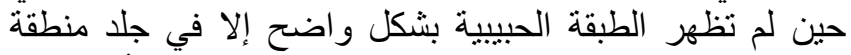
السطح البطني للإليه ومنطقة الصفن. كان للموسم تأثير على فلى

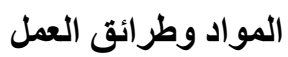

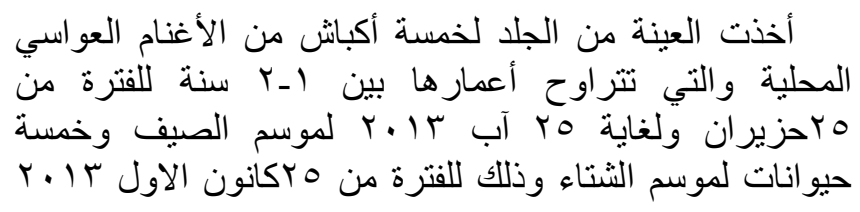




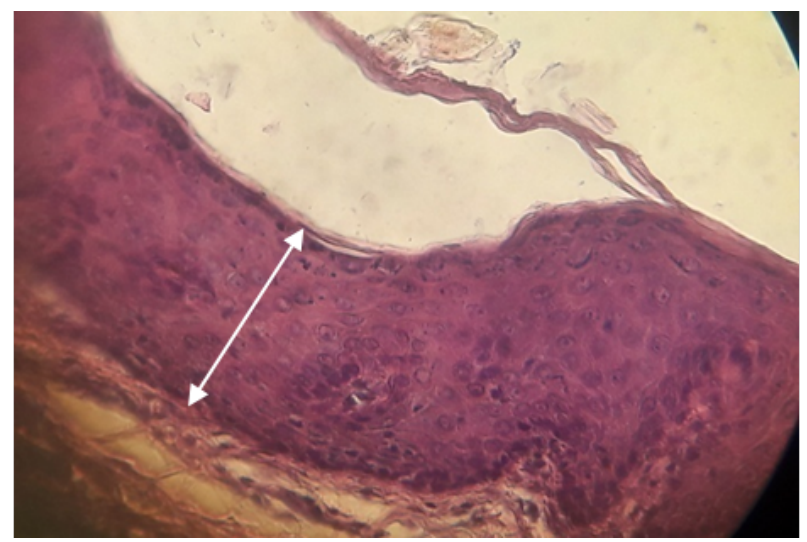

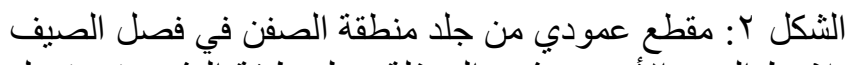

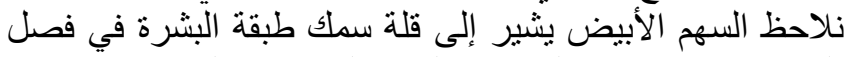

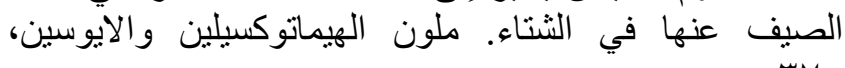

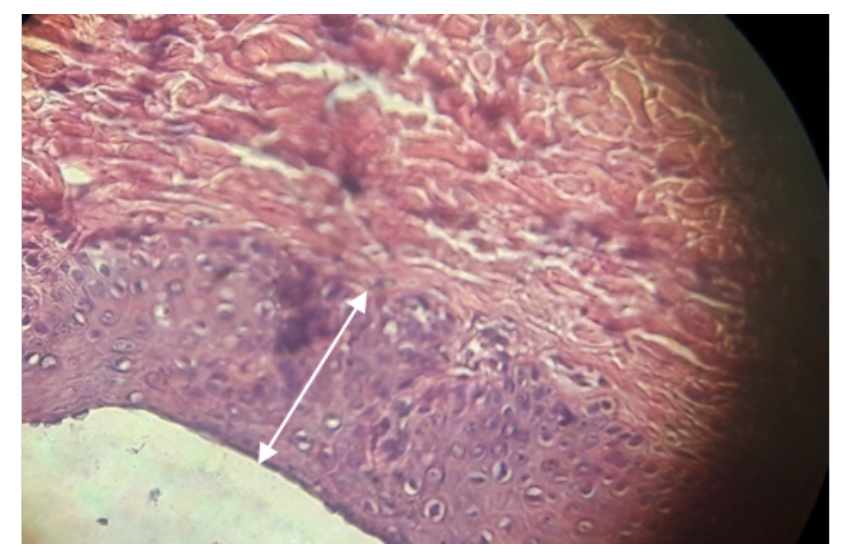

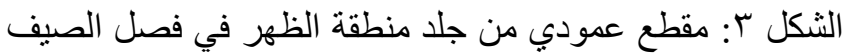

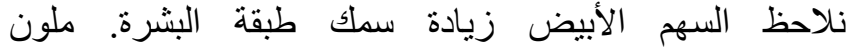

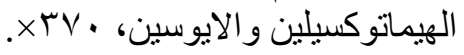

لم يلاحظ في دراستنا الحالية فروقا معنوية في أحجام الغدد

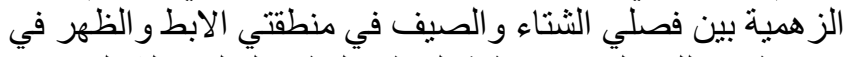

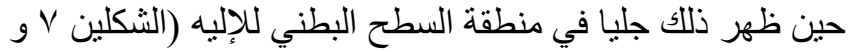

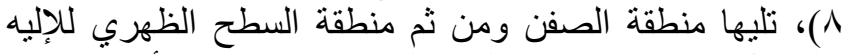

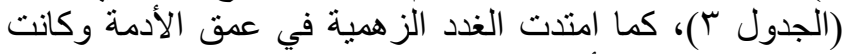

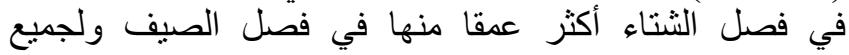

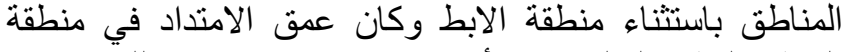

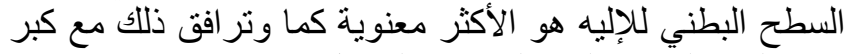

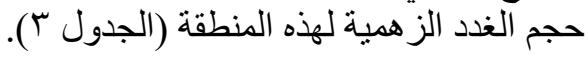

سمك طبقات الجلد و غدده العرقية والزهية حيث بينت الدر اسة

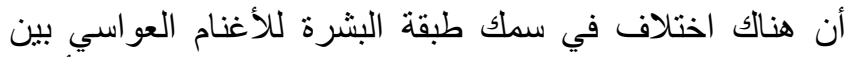

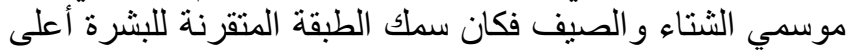

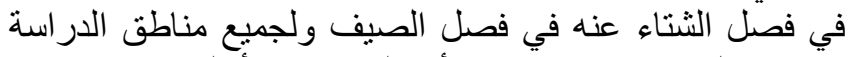

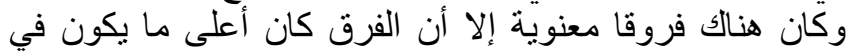

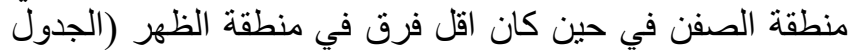

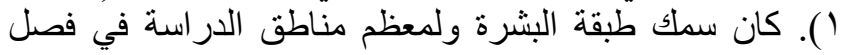

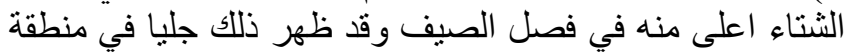

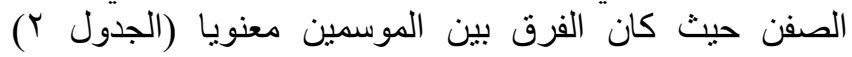

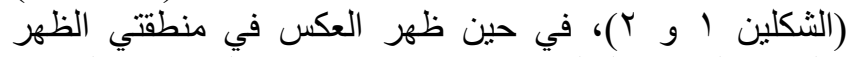

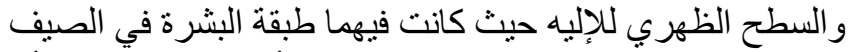

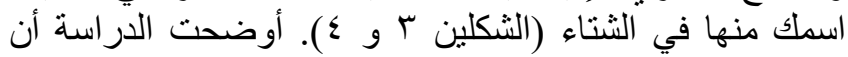

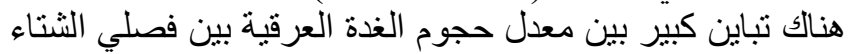

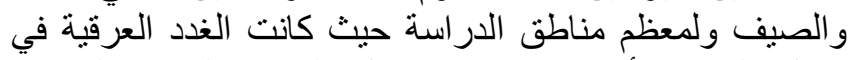

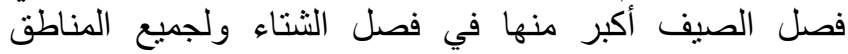
(الجدول ץ)، باستثناء منطقتي الصفن وجلد السطح البطني للإليه

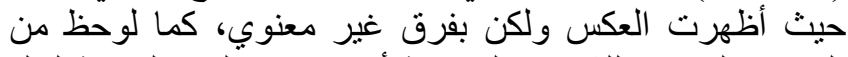

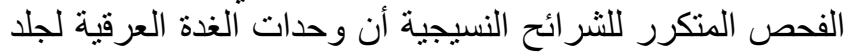

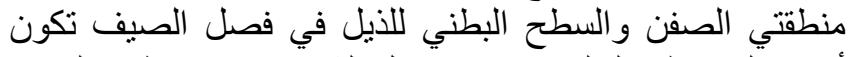

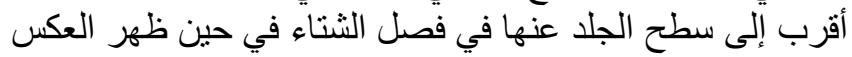

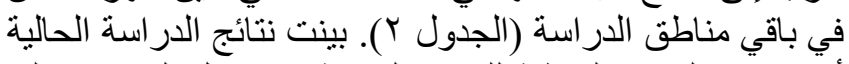

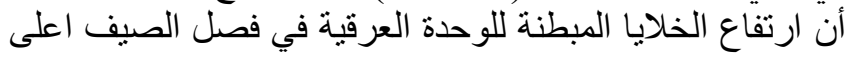

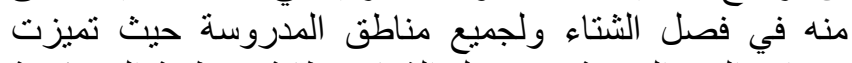

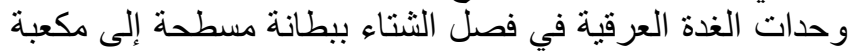

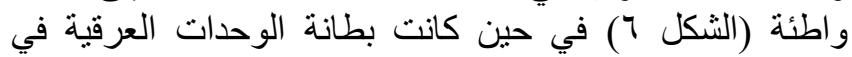

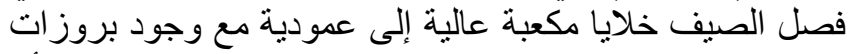

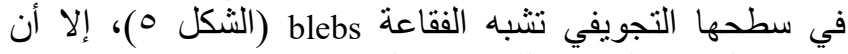

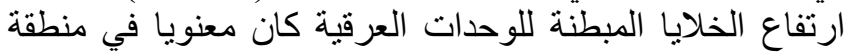
الصفن في فصل الصيف عنه في فصل الثتاء (الجدول كَ).

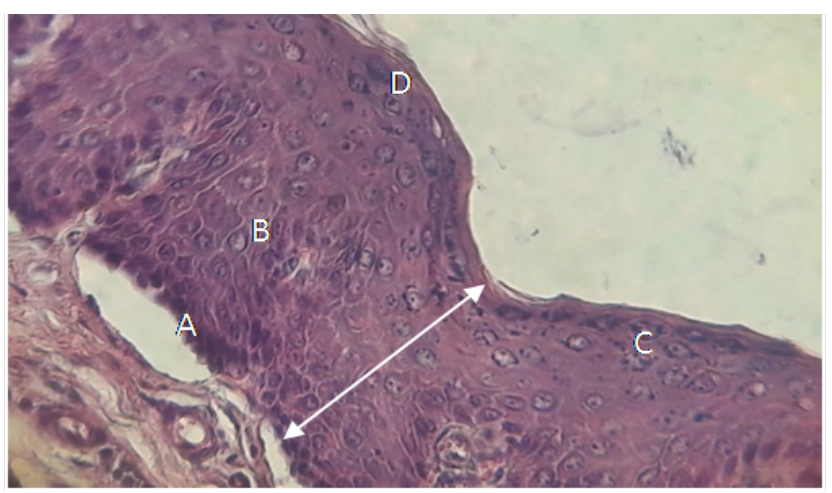

الشكل (: مقطع عمودي من جلد منطقة الصفن في فصل الثتاء

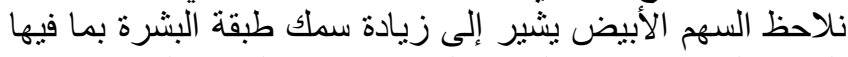

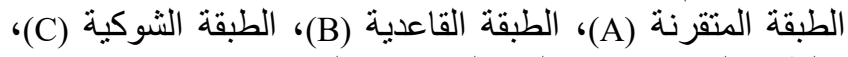

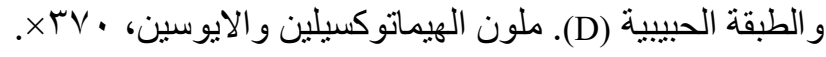


الجدول 1: يبين سمك الطبقة المتقرنة وسمك البشرة في المناطق التشريحية المختلفة من جلد الأغنام العواسي بين الثتاء و الصيف باستخدام وحدة قياس المايكروميتر (M+SE)

\begin{tabular}{|c|c|c|c|c|c|c|c|c|c|c|}
\hline \multicolumn{2}{|c|}{ السطح الظهري للإليه } & \multicolumn{2}{|c|}{ السطح البطني للإليه } & \multicolumn{2}{|c|}{ الصفن } & \multicolumn{2}{|c|}{ الظهر } & \multicolumn{2}{|c|}{ الابط } & المناطق \\
\hline صيف & شتاء & صيف & شتاء & صيف & شتاء & صيف & شتاء & صيف & شتاء & الموسم المتغير \\
\hline
\end{tabular}

سمك الطبقة

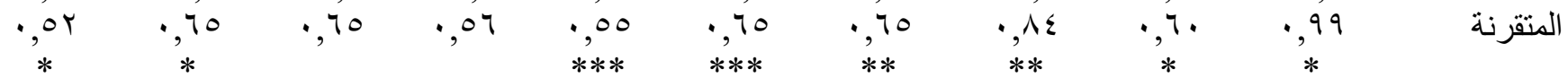

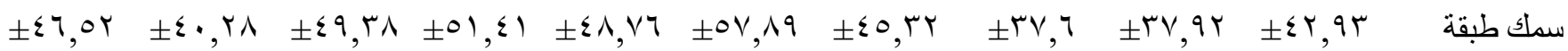

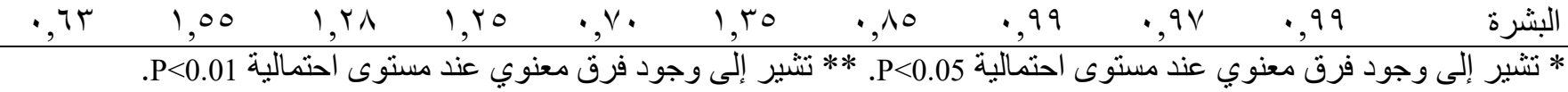

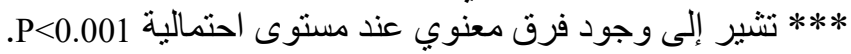

الجدول r : يبين قياسات الغدد العرقية في المناطق التشريحية المختلفة من جلد الأغنام العواسي بين فصلي الثتاء و الصيف باستخدام وحدة قياس المايكروميتر (MدSE)

\begin{tabular}{|c|c|c|c|c|c|c|c|c|c|c|}
\hline \multicolumn{2}{|c|}{ السطح الظهري } & \multicolumn{2}{|c|}{ السطح البطني للإليه } & \multicolumn{2}{|c|}{ الصفن } & \multicolumn{2}{|c|}{ الظهر } & \multicolumn{2}{|c|}{ الابط } & \multirow{2}{*}{ الموسم المتغير } \\
\hline صيف & شتاء & صيف & شتاء & صيف & شتاء & صيف & شتاء & صيف & شتاء & \\
\hline $\begin{array}{c} \pm V, r \wedge \\
r, \wedge\end{array}$ & $\begin{array}{c} \pm 0,91 \\
1,7\end{array}$ & $\begin{array}{l} \pm T, r r \\
r, r\end{array}$ & $\begin{array}{c} \pm 7, \leq \leq \\
1,9\end{array}$ & $\begin{array}{c} \pm \uparrow, r \\
1, r \\
*\end{array}$ & $\begin{array}{l} \pm T, r Y \\
1, Y \\
*\end{array}$ & $\begin{array}{l} \pm V, 1 T \\
1, \varepsilon\end{array}$ & $\begin{array}{c} \pm 7, \leq 0 \\
1,9\end{array}$ & $\begin{array}{c}* \\
\pm \wedge, q 1 \\
r, \varepsilon \\
*\end{array}$ & $\begin{array}{c}* \\
\pm 0,7 V \\
1, V \\
*\end{array}$ & حجم الغدة \\
\hline $\begin{array}{l} \pm \leqslant \wedge 0 \\
10, \leqslant \wedge\end{array}$ & $\begin{array}{l} \pm \varepsilon r \tau \\
1 \wedge, r .\end{array}$ & $\begin{array}{l} \pm 0 r \wedge \\
r, 1 \varepsilon\end{array}$ & $\begin{array}{l} \pm \leqslant \varepsilon 0 \\
17,97\end{array}$ & $\begin{array}{l} \pm 0{ }_{0} \\
r \leqslant, 01\end{array}$ & $\begin{array}{l} \pm r V . \\
10, V \leq\end{array}$ & $\begin{array}{l} \pm 0 \leqslant 0 \\
11, r_{0}\end{array}$ & $\begin{array}{l} \pm 00 . \\
r \cdot, \leqslant r\end{array}$ & $\begin{array}{l} \pm T r . \\
r \cdot, \varepsilon .\end{array}$ & $\begin{array}{l} \pm 017 \\
r r, \lambda .\end{array}$ & طول الغدة \\
\hline $\begin{array}{l} \pm \| \Lambda \\
1, \wedge 1 \\
* *\end{array}$ & $\begin{array}{l} \pm|r| \\
10, Y \leq \\
* *\end{array}$ & $\begin{array}{l} \pm I Y Y \\
\wedge, T Y \\
* * *\end{array}$ & $\begin{array}{l} \pm 1 r 4 \\
1 \cdot, V T \\
* * *\end{array}$ & $\begin{array}{l} \pm 1 r . \\
\vee, T . \\
* * *\end{array}$ & $\begin{array}{l} \pm 1 \leqslant 1 \\
9,79 \\
* * *\end{array}$ & $\begin{array}{l} \pm 1 \text { Y } \\
7,00 \\
* * *\end{array}$ & $\begin{array}{l} \pm I Y r \\
\wedge, q V \\
* * *\end{array}$ & $\begin{array}{l} \pm 1 r 0 \\
9, \leqslant 9 \\
* * *\end{array}$ & $\begin{array}{l} \pm 111 \\
9,79 \\
* * *\end{array}$ & قطر الغدة \\
\hline $\begin{array}{c}90 \cdot, \varepsilon \\
\pm \\
\mid \vee, 91\end{array}$ & 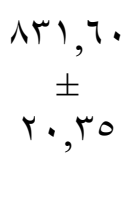 & $\begin{array}{c}r q \leq, r . \\
\pm \\
17, \leq 0 r\end{array}$ & $\begin{array}{c}V V Y, Y . \\
\pm \\
19,09\end{array}$ & $\begin{array}{c}\text { rv,.. } \\
\pm \\
1 r, \pi r \\
*\end{array}$ & $\begin{array}{c}T r 1, \cdots \\
\pm \\
10,90 \\
*\end{array}$ & $\begin{array}{c}\text { rso, }, \\
\pm \\
r r, 0 . \\
*\end{array}$ & $\begin{array}{c}q \cdot v, r \cdot \\
\pm \\
r \varepsilon, r \cdot \\
*\end{array}$ & $\begin{array}{c}117, \varepsilon \cdot \\
\pm \\
r \leqslant, 99 \\
* * *\end{array}$ & $\begin{array}{c}\varepsilon \varepsilon \cdot, r \\
\pm \\
|\wedge, r| \\
* * *\end{array}$ & الغداية ظهور \\
\hline $\begin{array}{c}\mid r \leq r, \varepsilon . \\
\pm \\
\mid \wedge, \wedge .\end{array}$ & $\begin{array}{c}\text { IrVo, } \\
\quad \pm \\
\text { ro,Ar }\end{array}$ & $\begin{array}{c}M v, \wedge . \\
\pm \\
r r, Y .\end{array}$ & $\begin{array}{c}1177, \varepsilon . \\
\pm \\
\Gamma \leq, 9\end{array}$ & $\begin{array}{c}11,1,7 \\
\pm \\
r q, \wedge r \\
* *\end{array}$ & $\begin{array}{c}q r q, 7 . \\
\pm \\
r \wedge, r r \\
* *\end{array}$ & $\begin{array}{c}\text { I } \vee \vee T, 7 . \\
\pm \\
\text { ro, }, 0 .\end{array}$ & $\begin{array}{c}1 \leq 90, \wedge . \\
\pm \\
r \cdot, \wedge\end{array}$ & $\begin{array}{c}\text { lor. } ., \\
\pm \\
r 0, r \\
*\end{array}$ & $\begin{array}{c}097, \wedge . \\
\pm \\
11, r q \\
*\end{array}$ & الغراية ظهور \\
\hline $\begin{array}{c}M Y, Y \varepsilon \\
\pm \\
\cdot, T V\end{array}$ & $\begin{array}{c}11,99 \\
\pm \\
., 01\end{array}$ & $\begin{array}{l}17, \Gamma \leqslant \\
\pm \\
\cdot, \wedge \wedge\end{array}$ & $\begin{array}{c}1 \leq, 71 \\
\pm \\
\cdot, 7 .\end{array}$ & $\begin{array}{l}17, \leqslant r \\
\pm \\
\cdot, \wedge 9\end{array}$ & $\begin{array}{c}1 \cdot, \cdot V \\
\pm \\
\cdot, 99\end{array}$ & $\begin{array}{c}11,9 \leq \\
\pm \\
\cdot, T \leq\end{array}$ & $\begin{array}{l}11,7 \\
\pm \\
., 09\end{array}$ & $\begin{array}{c}11,00 \\
\pm \\
\cdot, 11\end{array}$ & $\begin{array}{l}9, \cdot 1 \\
\pm \\
\cdot, 7 \leq\end{array}$ & العرقية الغدة \\
\hline
\end{tabular}


الجدول r: يبين قياسات الغدد الزهمية في المناطق التشريحية المختلفة من جلد الأغنام العو اسي بين الثنتاء و الصيف باستخدام وحدة قياس

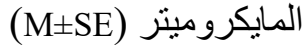

\begin{tabular}{|c|c|c|c|c|c|c|c|c|c|c|}
\hline \multicolumn{2}{|c|}{ السطح الظهري للإليه } & \multicolumn{2}{|c|}{ السطح البطني للإليه } & \multicolumn{2}{|c|}{ الصفن } & \multicolumn{2}{|c|}{ الظهر } & \multicolumn{2}{|c|}{ الابط } & \multirow{2}{*}{ الموسم المتغير } \\
\hline صبف & شتاء & صيف & شتاء & صبف & شتاء & صيف & شتاء & صيف & شتاء & \\
\hline$* *$ & $* *$ & $* * *$ & $* * *$ & * & * & & & & & \\
\hline $1 \cdot 1$, & lor. & 1.7, & ¿ı,. & $1 \cdot \varepsilon$, & Ir, & 117 & $1 \leqslant \cdot$, & Ir., & $11 \leqslant$, & البعد القصير \\
\hline \pm & \pm & \pm & \pm & \pm & \pm & \pm & \pm & \pm & \pm & الغدد الزهية \\
\hline$r, v \varepsilon$ & 9,9 & $\Lambda, I r$ & $10, \mu$ & $\Lambda, Y \mu$ & 9,79 & $1 \wedge$ & $17,0$. & $\varepsilon, \vee q$ & $\vee, \leq \wedge$ & \\
\hline$*$ & $*$ & $* * *$ & $* * *$ & $* *$ & $* *$ & & & $*$ & * & \\
\hline IV., & ros & 10. & $\uparrow \wedge \cdot$, & $1 \leq 7$, & $r \varepsilon \cdot, \cdot$ & YrT, & lAr, & $r r \varepsilon$, & $r \leqslant \wedge$, & البعد الطويل \\
\hline \pm & \pm & \pm & \pm & \pm & \pm & \pm & \pm & \pm & \pm & الغدد الز همية \\
\hline$i r, \wedge \wedge$ & $1 v,{ }_{10}$ & $7,{ }^{2} 0$ & דו, 11 & $9, Y V$ & $1 \cdot, \leqslant \wedge$ & ir, & $11, \leqslant 1$ & V,Ar & $q, \vee \leqslant$ & \\
\hline & & $* * *$ & $* * *$ & $* *$ & $* *$ & $* *$ & $* *$ & $*$ & $*$ & \\
\hline$\leqslant 10, \wedge$. & $\varepsilon r r, \ldots$ & 179,7 & $\mu q 9,7$. & $17 V, \varepsilon$. & $r \wedge \cdot, \cdots$ & $r / 7, \cdots$ & rqv, . & $19 \cdot, 7$ & $101, r$. & بداية ظهور \\
\hline \pm & \pm & \pm & \pm & \pm & \pm & \pm & \pm & \pm & \pm & الغدد الزهمية \\
\hline $9,0 r$ & 10,17 & 10,17 & $r v, T$. & $10, V \varepsilon$ & $I V, V \varepsilon$ & 9,9 & $\Lambda, r$. & $1 ., 1$. & $1 \cdot, \wedge$. & \\
\hline & & $* * *$ & $* * *$ & $* *$ & $* *$ & $* * *$ & $* * *$ & $*$ & $*$ & \\
\hline$O \wedge \Lambda$ & $01 \pi, .$. & $r \cdot r, \varepsilon \cdot$ & Tor, $\varepsilon$. & $r \cdot r, \varepsilon$. & sor,, . & TVY,T. & $O r \leqslant, 7$. & $r ו, r$. & rvo, $\varepsilon$. & نهاية ظهور \\
\hline \pm & \pm & \pm & \pm & \pm & \pm & \pm & \pm & \pm & \pm & الغدد الزهمية \\
\hline 17,11 & 10,71 & 11,11 & ru,q. & $11, \ldots$ & $r r, 1 \cdot$ & $10, V \varepsilon$ & $1 v, 91$ & $1 \leq, 71$ & $1 \cdot, 1 \mathrm{~V}$ & \\
\hline
\end{tabular}

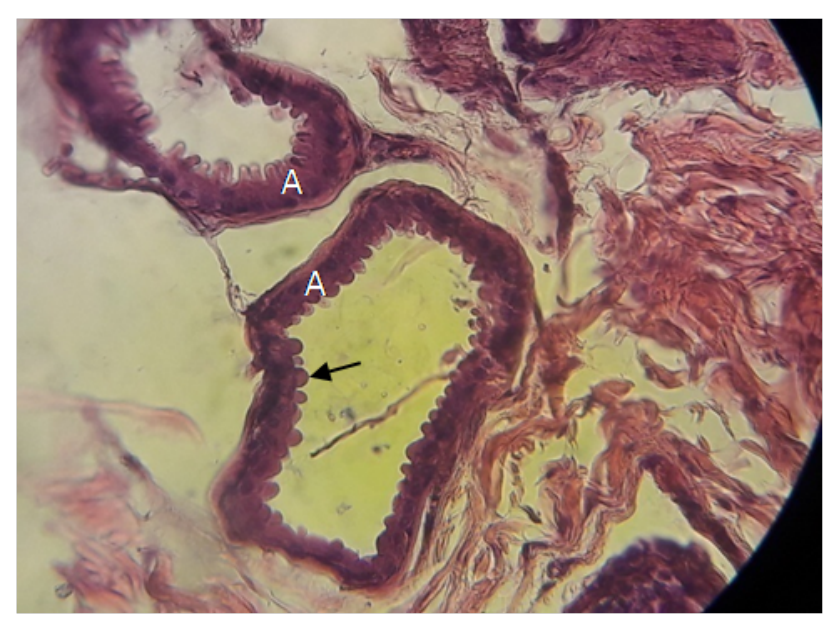

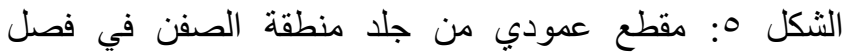
الصيف، إذ يلاحظ بين الوحدة الإفرازية العرقية ارتفاع خلايا

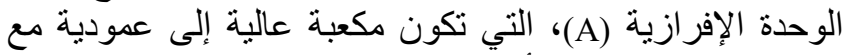

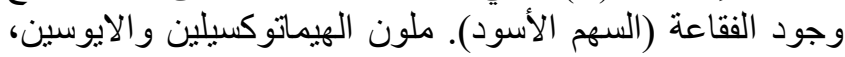

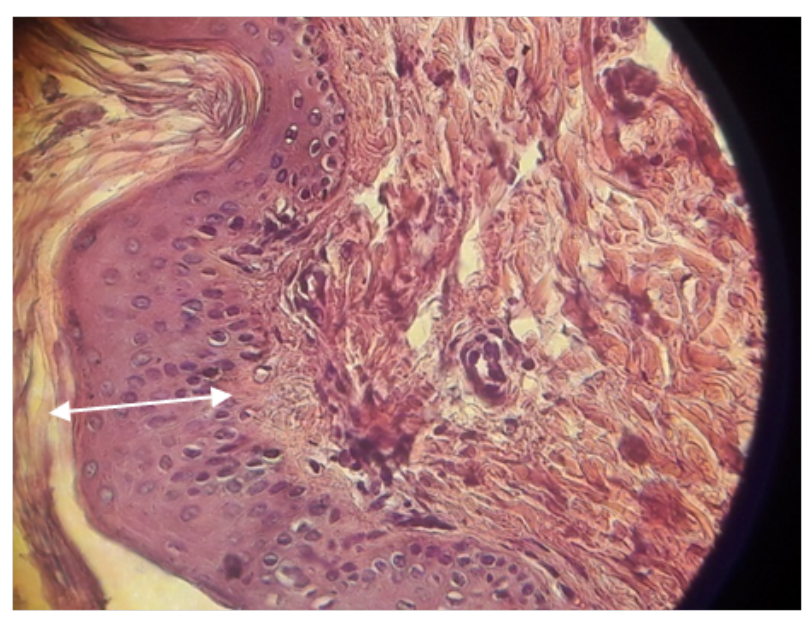

الشكل §: مقطع عمودي من جلد منطقة الظهر في فصل الشتاء

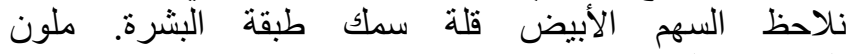

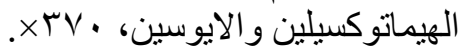




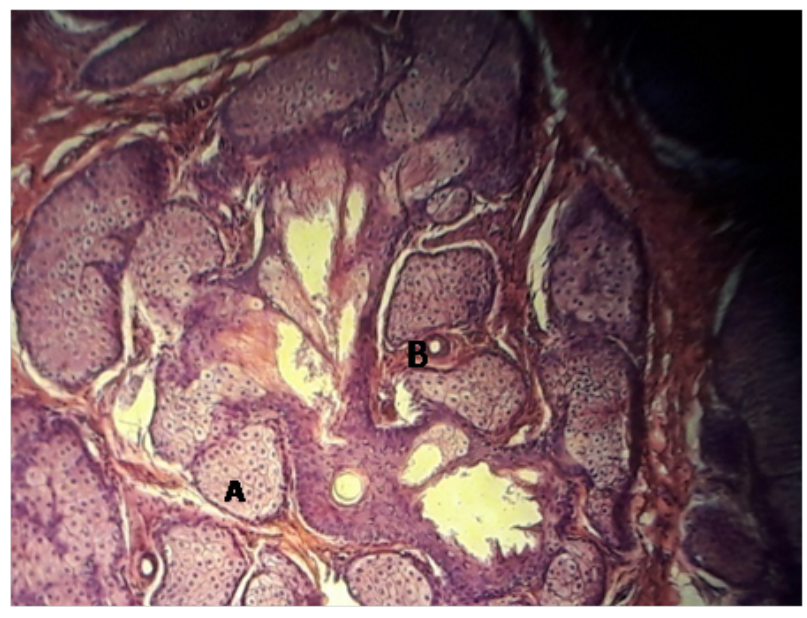

الثكل ^: مقطع أفقي من جلد منطقة السطح البطني للإليه في

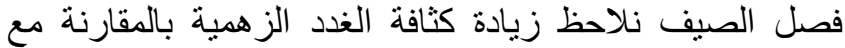

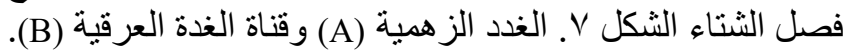

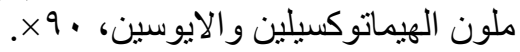

فصلي الثتاء و الصيف، حيث كان سمك طبقة البشرة ولمعظم

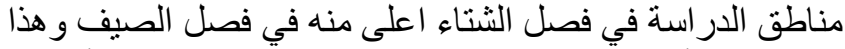

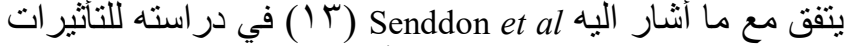

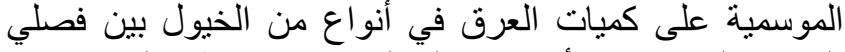

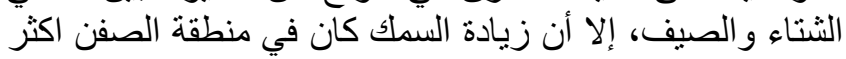

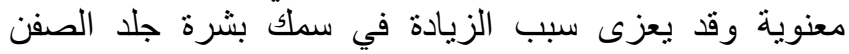

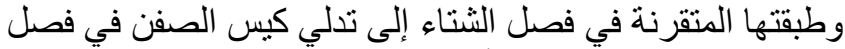

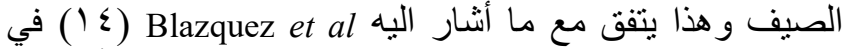
دراسته لعدة مناطق لجلد الصفن في الثيران، في حين أظهرت

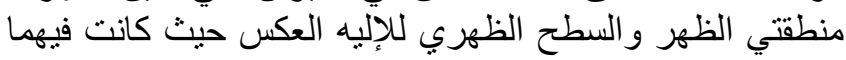

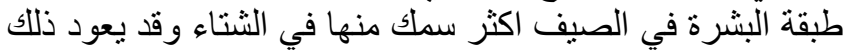

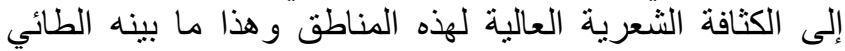

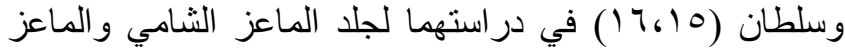

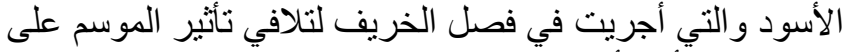

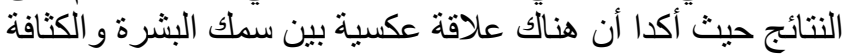

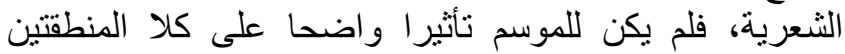

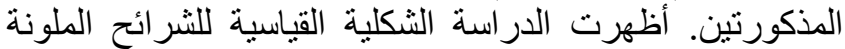

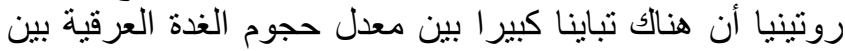

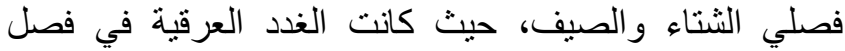
الصيف اكبر منها في فصل الشتاء ولمعظم مناطق الداء الداء

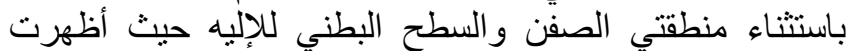

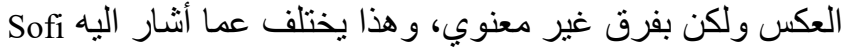

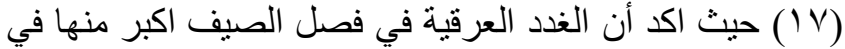

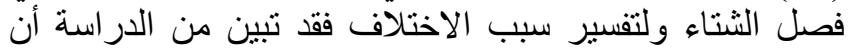

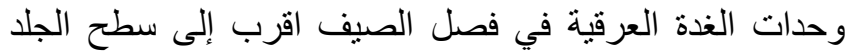
منها في فصل الثتاء في كلا المنطقتين (جلد الصفن والسطح

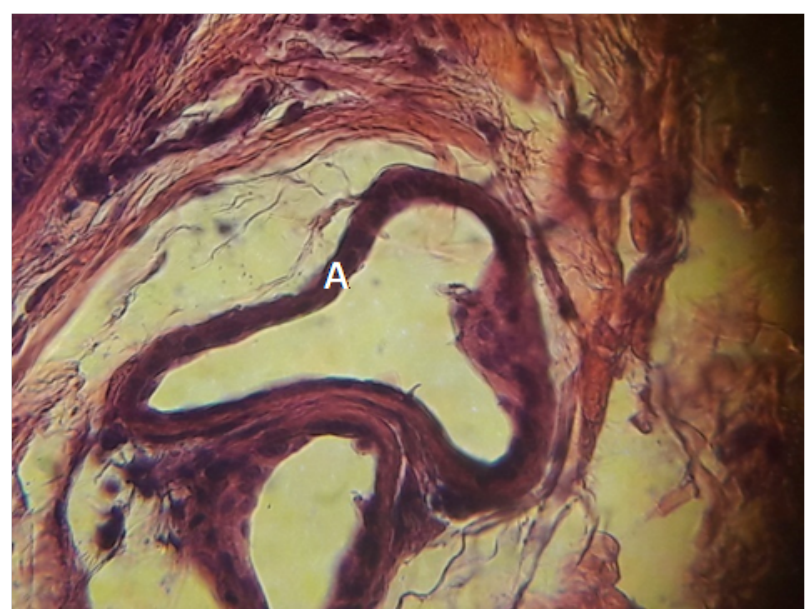

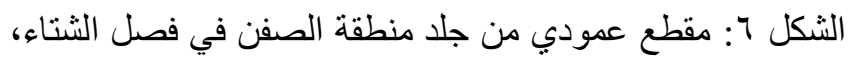

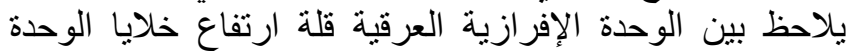

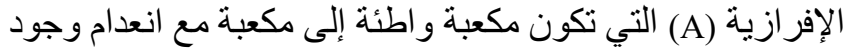

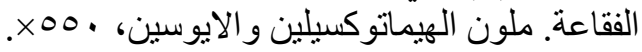

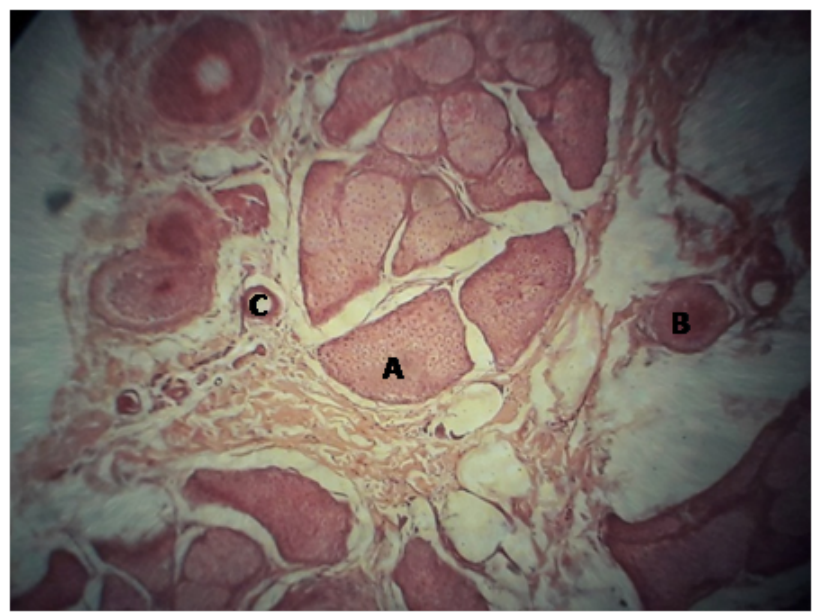

الثكل V: مقطع أفقي من جلد منطقة السطح البطني للإلبه في

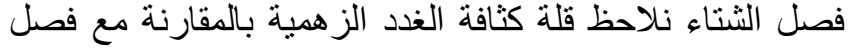

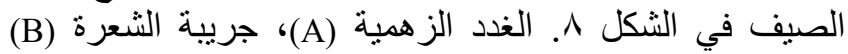

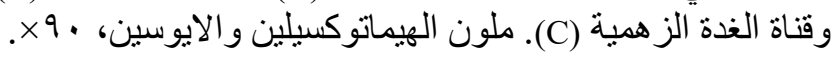

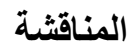

لم تبين الدر اسة وجود اختلاف في التركيب النسيجي لجلد الأغنام

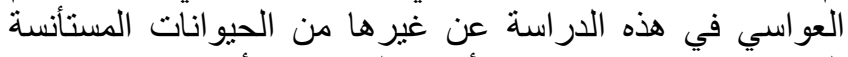

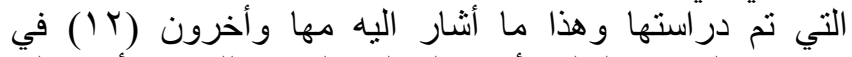

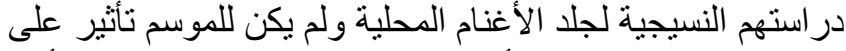

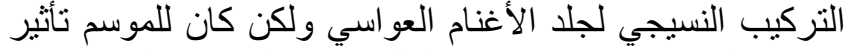

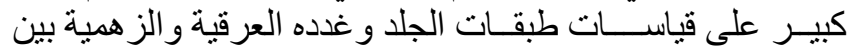


6. Al-Umeri SKW, Al-Mamoori NAM. Comparative histological and histochemical study of flank region skin, in camel, cow and buffalo. Al-Qadisiya J Vet Med Sci. 2016;15(2):102-107.

7. Mobini B. Effect of age and sex on fiber and follicle characteristics of an Iranian native sheep. Socoto J Vet Sci. 2012;10(1):1-4. http://dx.doi.org/10.4314/sokjvs.v10i1.1

8. Abdou ASA, Guirgis RA, El-Ganaieny MM. Some Histological changes in the hair and skin follicles of dromedary camels at different ages. Alex J Agric Res. 2006;51(2):17-26.

9. Luna LG. Manual of histological staining methods of the armed forces in statute of pathology. $3^{\text {rd }}$ ed. New York: McGraw Hill back Company; 1968. 12-18 p.

10. Culling CFA, Allison RT, Barr WT. Cellular pathology technique. $4^{\text {th }}$ ed. Butterworth: CRC Press; 1985. 6, 167 p.

11. Jenkinson DM, Nay T. Sweat gland and hair follicle measurements as indicators of skin type in cattle. Aust J Biol Sci. 1968;21(5):10011011.

T ا. مها عبد الهادي، نجاح هاثم المحنة، عدنان وحيد البديري. در اسة نسيجية لجلد الأغنام المحلية Ovis ovis. مجلة هائة القادسية لعلوم الطب البيطري.

$$
\text { .19_1 } \leqslant:(1) 1 \leq \leqslant .10
$$

13. Senddon JC, Ritruechai P, Yanes GS, Howard V. Seasonal influences on quantitative changes in sweat-associated anatomy in native and thoroughbred horses. ESVD and ACVD. 2008;19:163-173. https://doi.org/10.1111/j.1365-3164.2008.00671.x

14. Blazquez NB, Mallard GJ, Wedd SR. Sweat glands of the scrotum of the bull. J Rerprod Fertil. 1988;83(2):673-677. doi:10.1530/jrf.0.0830673

1 ـ الطائي احمد نزار. دراسة طبو غر افية نسيجية مقارنة لجلد الماعز الثامي

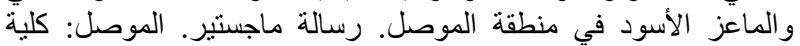

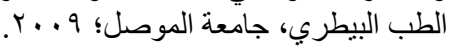

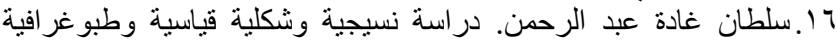

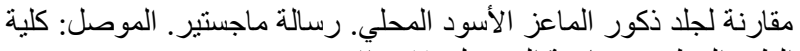

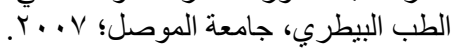

17. Sofi SA. Histological study of scrotum in rams. Res Opin Anim Vet Sci. 2012;2(8):459- 463.

18. Amakiri SF, Adepoju JJ. Changes in sweat gland morphology in cattle before and during heat stimulation. Acta Anat. 1979;105:140-150. doi; $10.1159 / 000145118$

19. Thody A J, Shuster S. Control and function of sebaceous glands. Physiol Rev. 1989;69(2):383-416. doi:10.1152/physrev. 1989.69.2.383

20. Dyce KM, Sack WO, Wensing CJG. Text book of veterinary anatomy. $3^{\text {rd }}$ ed. USA: WB Saunders; 2004. 811 p.
البطني للإليه) في حين كان عمث وحدات الغدد العرقية في باقي

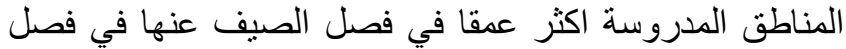
الثتاء وبفروق معنوية. كما لوحظ أن ارتفاع الخلايا المبطنة للوحدة العرقية في فصل الصيف كان اعلى منه في فصل الثتاء

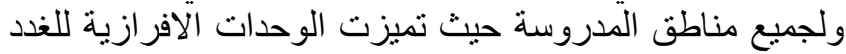
العرقية في فصل الصيف بوجود بروزات في سطحها التجويفي تشبه الفقاعة blebs فضلا عن ارتفاع خلاياها المبطنة للوحدة الافرازية في حين كانت بطانة الوحدات العرقية في فصل الثتاء

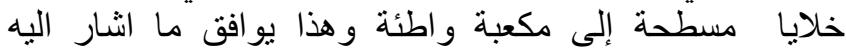
Amakiri

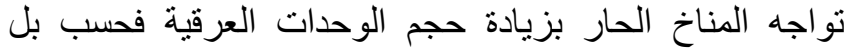

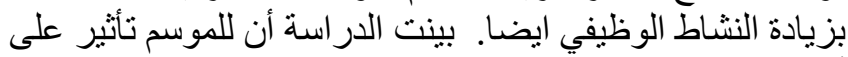
أحجام الغدد الزهية في منطقة السطح البطني للإليه ومنطقة الزية الصفن أكثر من باقي المناطق وقد يكون السبب وجود العئ الكميات العالية من الدهون في منطقة الإليه حيث أشار

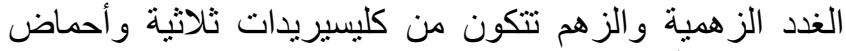
دهنية حرة، أما منطقة الصفن فقد يعود إلى دور جلد الصن الصفن في في المحافظة على درجة الحرارة المناسبة للحيامن في فصل الثناء الثناء

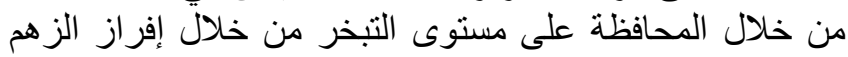
(Y.)

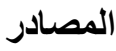

1. Mescher AL. Junqueira's basic histology textbook and atlas. $14^{\text {th }}$ ed. New York: McGraw-Hill education;2016. 372-381p.

2. El-shafey AA, Emam MA, Kassab AA. Histomorphometric and immunohistochemical characteristics of the skins of egyptian water buffalo (Bubalus bubalis) and one-humped camel (Camelus dormedarius). J Vet Anat. 2017;10(1):1-16.

3. Hekal SA. Histological study of the skin and leather characteristics in two types of arabian camels (Camelus dormedarius). J Am Sci. 2014;10(9):41-47. doi:10.7537/marsjas100914.06

4. Obeyes AK. Histological study for skin of Horse. Tikrit J Pu Sci. 2016;21(1):31-35.

5. Ibrahim RS, Hussein AA, Jabbar AJ. Comparative microscopically study of the skin in local and wild rabbits. Kufa J Vet Med Sci. 2017;8(1):151-165. 